\title{
Spontaneous rotations in epithelia as an interplay between cell polarity and RhoA activity at boundaries
}

\author{
Lo Vecchio S. ${ }^{1-5}$, Pertz O. ${ }^{9}$, Szopos M. ${ }^{8}$, Navoret L. ${ }^{6,7 *}$, Riveline $\mathrm{D}^{1-5 *}$ \\ ${ }^{1}$ Laboratory of Cell Physics ISIS/IGBMC, CNRS and University of Strasbourg, \\ Strasbourg, France. \\ ${ }^{2}$ Institut de Génétique et de Biologie Moléculaire et Cellulaire, Illkirch, France. \\ ${ }^{3}$ Centre National de la Recherche Scientifique, UMR7104, Illkirch, France. \\ ${ }^{4}$ Institut National de la Santé et de la Recherche Médicale, U964, Illkirch, France \\ ${ }^{5}$ Université de Strasbourg, Illkirch, France. \\ ${ }^{6}$ Institut de Recherche Mathématique Avancée, UMR 7501, Université de Strasbourg \\ et CNRS, France \\ ${ }^{7}$ INRIA Nancy-Grand Est, TONUS Project, Strasbourg, France \\ ${ }^{8}$ Laboratoire MAP5, UMR 8145, Université de Paris et CNRS \\ ${ }^{9}$ Cellular Dynamics Lab, Institute of Cell Biology, Bern University \\ * Co-corresponding authors. Contacts: \\ laurent.navoret@math.unistra.fr and riveline@unistra.fr
}

\begin{abstract}
Directed flows of cells in vivo are essential in morphogenesis. They shape living matter in phenomena involving cell mechanics and regulations of the acto-myosin cytoskeleton. However the onset of coherent motion during collective cell migration is still poorly understood. Here we show that coherence is set by spontaneous alignments of cell polarity by designing cellular rings of controlled dimensions. A tugof-war between opposite polarities dictates the onset of coherence, as assessed by tracking live cellular shapes and motions in various experimental conditions. In addition, we identify an internally driven constraint by cellular acto-myosin cables at boundaries as essential to ensure coherence and active force is generated as evaluated by the high RhoA activity. Its contribution is required to trigger coherence as shown by our numerical simulations based on a novel Vicsek-type model including free active boundaries. Altogether, spontaneous coherent motion results from basic interplay between cell orientations and active cables at boundaries.
\end{abstract}

\section{INTRODUCTION}

Morphogenesis is driven by the interplay between Rho GTPases and the cytoskeleton ${ }^{1-4}$. In particular, the acto-myosin network drives morphogenesis at the cellular level by elongation or neighbor exchange ${ }^{5,6}$, and the cross-talk between Rho signaling and cellular mechanics has emerged as a generic property of morphogenetic systems. Interestingly, these dynamics are often associated with directed motions of cells. Directional flows in epithelia are reported during development in a variety of systems ranging from Drosophila to zebrafish ${ }^{7-9}$. For example, during the development of spherical mammary acini or during the egg chamber elongation in Drosophila, cells exhibit coherent rotation on large scales with speed up to $20 \mu \mathrm{m} \cdot \mathrm{h}^{-1}{ }^{10-13}$. These motions are proposed to be important for the robust morphogenesis of embryos. 
The collective motion of cells is difficult to understand based on a simple analysis of migrations at the single cell level. Individually, cells can move randomly or directionally. However the epithelial layers which connect cells through adherens junctions may or may not coordinate the motion of the group ${ }^{14-16}$. The understanding of emergence of collective effects requires theoretical approaches in tight comparison with experiments. In addition, although the interplay between RhoA activity and the acto-myosin cytoskeleton is documented at the single cell level ${ }^{1,17,18}$, it is not yet fully characterized at the multi-cellular scale. In this context of potential collective effects, the understanding of coherent motion requires to study cellular assembly in simple geometries in vitro compared quantitatively to numerical simulations.

To this aim, we generated rings of epithelial cells, a geometry proposed by Turing in his 1952 article ${ }^{19}$. This initial condition led to the formation of rings of connected cells, a simple and reproducible periodic configuration. We varied the ring diameter to identify the inherent coherence length of our epithelial MDCK cells. We report that below a threshold perimeter, rings undergo spontaneous rotations. We next sought to extract the ingredients generating this coherence. We show that an internal tug-ofwar between cell polarities within the ring determines the onset of coherence, as shown by the initial polarity distributions and the time required to reach coherence. Tracking of cell division as polarity breaker supports this framework. We also report that coherence is associated with two supracellular acto-myosin cables at the inner and outer boundaries of the monolayer: these continuous structures act as autonomous confinements as they exhibit high RhoA activity and are shown to prevent cells from spreading out and breaking coherence. Finally, we test our central mechanism of polarity tug-of-war in silico with a Vicsek-based model writing the fundamental laws of dynamics at cellular levels for polarity, velocity and including forces at boundaries. We show from this computational approach that dynamics of all rings are reproduced quantitatively and that activities at boundaries - supported by RhoA activity in experiments - is indispensable to ensure cellular coherence. We propose that cell polarity tug-of-war and confinement from spontaneously assembled active cables set the tempo for coherent movements in epithelial monolayers.

\section{RESULTS}

\section{Obtaining coherent motion}

We first needed to identify the natural length over which cells migrate directionally namely the coherence length $\xi_{\max }$ - characterizing our system. A circular array of cells is expected to undergo rotation if its perimeter, $p$, matches the maximal coherence length $\xi_{\max }$. If the coherence length is smaller than the perimeter, noncoherent motion is expected ${ }^{20,21}$. To obtain $\xi_{\max }$, we designed multicellular rings with decreasing diameters (see Methods) : $1000 \mu \mathrm{m}, 300 \mu \mathrm{m}$ and $180 \mu \mathrm{m}$ (see Movies S1 to S3, respectively), and we measured the 12-hours velocity fields (see Methods). Rings had different behaviors: large rings - 1000 $\mu \mathrm{m}$ - exhibited growth of cellular fingers inwards and outwards whereas smaller rings - 300 $\mu \mathrm{m}$ and $180 \mu \mathrm{m}$ - behaved like cellular assemblies during wound healing with centripetal closure (see Fig.1a and Movies S1-S3). We saw coherent flows of length $\xi$ (Fig. 1b) and no global coherence was obtained for $1000 \mu \mathrm{m}$ and $300 \mu \mathrm{m}$ rings. However, about a quarter of rings spontaneously rotated at $180 \mu \mathrm{m}$ diameter with a velocity of $\sim 20 \mu \mathrm{m} . \mathrm{h}^{-1}$, which suggested that we approached the perimeter value potentially leading to coherent 
motion. To find this perimeter, we plotted the correlation function of tangential velocity, and we extracted the coherence length $\xi_{\max }$ (see Fig.1c, Fig.S1a and Methods) ${ }^{22}$. We show that $\xi_{\max }$, obtained from the $\mathrm{x}$-intersect of the tangent at origin of the correlation function, is similar in every condition $-\xi_{\max }=315 \mu \mathrm{m}$ (Fig. 1c and Fig. S1a) -, demonstrating that $\xi_{\max }$ is an inherent length of our cellular system.

Based on this $\xi_{\max }$ of $315 \mu \mathrm{m}$, we designed and focused on rings with $80 \mu \mathrm{m}$ diameter corresponding to $250 \mu \mathrm{m}$ perimeter below $\xi_{\max }$. As expected, we "rectified" the motion of cells around the ring and we ended up with $83 \%$ of rings spontaneously rotating at a velocity of 20-25 $\mu \mathrm{m}^{-1} \mathrm{~h}^{-1}$ (see Movie S4, Fig. 1d and Fig. S1b). All results are reported in Figure 1e, where $\frac{\xi_{\max }}{p}$, the coherence length over the perimeter, is defined as an index of global coherence: it is equal to 1 when the whole ring rotates and gets close to 0 as the global coherence decreases. Interestingly, there is a bias in clockwise rotation which suggests a spontaneous cellular bias as reported in previous works ${ }^{23,24}$ (Fig. S1c).

If coherence was obtained for this $80 \mu \mathrm{m}$ ring diameter, the time needed to reach this rotation varied from 1 hour to 10 hours. This large distribution impeded quantification of the transition from non-coherent to coherent motions. We decided to align all plots with respect to the onset of coherence defined as $\frac{\xi}{p}=1$. We could then determine the quantitative behavior of coherence acquisition. This pseudo-time representation allowed to show that coherence acquisition is a process lasting about 1 h30 with minor variability among rings (Fig. 1f). This timescale is fast considering the 12-hours duration of the experiment and this can be viewed as a quick switch between the two states. At coherence, two rings populations appeared: rings with persistent rotation over hours and rings with transient coherent motion shorter than one hour (Fig. If and Fig. S1d-e). To understand the origin of these two populations, we measured next the ring velocity and persistence of rotation, and saw their correlations (Fig. S1f). This suggests that collective cell speed might be involved in maintaining coherence even though inertia is negligible at this scale.

\section{Tug-of-war between cell polarities sets coherence}

Next we sought to identify the cellular mechanism at play for the onset of coherent rotation. Cell polarity is a natural readout for its key role in setting direction of motion. We thus looked at the distribution of cell polarities inside rings. For this, we used labelled lamellipodia (see Fig. 2a, see Methods). We observed selected lamellipodia orientation and direction. Initial polarities showed a bi-modal distribution peaked around tangential directions with two main orientations, i.e. $0^{\circ}$ and $180^{\circ}$ (see Fig. $2 \mathrm{~b}$ ). These two states suggest that a tug-of-war between polarities could happen between cells of opposite directions within the same ring. We reasoned that initial polarities could then play a role in the time needed to reach coherent rotation. A large number of cells with the same polarities would minimize the tug-of-war, and would decrease the time to coherence. We then followed each cell polarity defined by tight-junction organisation (see Fig. 2c and Movie S5). We had checked that the direction of shape anisotropy defined by cell geometry correlates with the direction of the lamellipodia (Fig. S2a-b). Our measurements of percentage of cells with aligned polarities as a function of time to coherence show a decreasing relationship (Fig. 2d). This confirms 
that distribution of single cell polarities imposes a tug-of-war and determines the time needed to enter coherence.

The co-existence of opposite polarities is expected to generate stretching within the ring. From tight junctions contours, we could also extract cell elongation (Fig. 2c and Fig. S2). Using the pseudo-time as the reference (Fig. 2e), we report three phases: (i) 2.5 hours before coherence, cells elongate until (ii) their tangential stretch reaches constant values sustained for about 1 hour; (iii) coherence starts shortly within $1 \mathrm{~h}$ followed by a rapid drop in elongation. These measurements substantiate our framework : cell lamellipodia "pull" in opposite directions leading to cellular stretch and resolution of this competition induces cell relaxation visualized by the drop in the stretch.

\section{Cell divisions as polarity breakers impede coherence}

To further test the central role for polarity in setting coherence, we tracked cell division events within rings. Right after mitosis, cells have opposite polarities and thus are expected to challenge polarity alignments. These opposite polarities indeed happened at cell division within rings (see Figure 2f). We quantified the effect by plotting the cumulative number of cell divisions within rings. We saw that rings where division regularly occurred were related to poorly coherent motion (Fig. $2 \mathrm{~g}$, bottom). Divisions generate cells with opposite polarities and impede coherence. In contrast, when a burst of many cell divisions occurred within a short time period (typically 2 hours over a total time of experiment of 12 hours), coherence was maintained in its optimal value (Fig. $2 g$, top). Division as a polarity breaker acts as a coherence breaker.

\section{Acto-myosin cables as internally self-assembled boundaries}

We reported that cells were polarized and we next sought to further understand why tangential orientation was selected. If the rings were confined by physical walls, this orientation would be favored ${ }^{25}$. However, in our assay, cells were free to move in any directions. So we stained for the inner and outer boundaries, since acto-myosin cables were reported to self-assemble within cells at epithelial colony boundaries ${ }^{17,26}$ (Fig. S3a). We show in Figure $3 a$ the presence of these acto-myosin cables. This suggests that cells internally set "walls" which in turn could potentially make them be oriented along the perimeter rather than radially.

We then evaluated the mechanical contributions of these cables and their roles in cell confinement. First with live observations, we saw that the inner cable was contractile and led first to the closure of rings within hours prior to extension 'out' of the cellular disk now formed (Fig. 3b and Movies S3-4). This suggests that indeed the inner cable pulls inwards as expected for a contracting acto-myosin cable. Then, we reasoned that the outer ring may act the same way by pushing inwards cells. To evaluate whether these structures were contractile, we used laser ablation experiments ${ }^{27}$. First we observed a fast and large opening of both cables within seconds with a similar extent of $\sim 12 \mu \mathrm{m}$ (see Figure 3c and 3d and Movie S6, see also Fig. S3b, and Methods). This demonstrates that cables are more tensile than cell-cell junctions also tested in these experiments which yielded small openings ( 
$1 \mu \mathrm{m})$. In addition, we measured the opening velocity after laser ablation as a readout for comparing stress between inner and outer cables assuming they bear the same damping coefficients ${ }^{27}$. Our results suggest that acto-myosin cables indeed apply contractile constraints on the boundary cells and with similar values (see Fig. 3d).

Next, we tested the impact of this contraction at boundaries on cell polarity. We followed cells transfected with Lifeact within non labeled rings. We report in Fig. $3 e$ and Movie S7 that cells could build their own acto-myosin cables while orienting their lamellipodia tangentially. This supports the notion that tension at cable and tangential polarity are correlated.

To further test this coupling, we sought to remove locally the acto-myosin cable. We included within rings single cells expressing caldesmon (CaD), a calmodulin binding protein inhibiting Myosin ATPase ${ }^{28}$. Indeed this triggered a local decrease in myosin activity and in cable contractility. These caldesmon expressing cells extended out of the rings and no rotations occurred in the majority of cases (see Fig. $3 f$ and Movie S8). On average, we found that coherence was lower on $\mathrm{CaD}$ mosaic rings (Fig. $3 \mathrm{~g}$ ) and this was correlated to the amount of CaD levels (Fig. S3c). This suggests that continuity of the acto-myosin rings is important for coherence. In conclusion, cables are essential to confine and orient cells polarities tangentially and further trigger rotations.

\section{High cell velocity and ring coherence correlate with low RhoA activity}

Observations and measurements of velocity and polarity could hide signaling effects associated to regulations of the acto-myosin cytoskeleton by the small GTPase RhoA. To evaluate this potential contribution, we analyzed the correlation between sought to correlate spatio-temporal dynamics of RhoA activity and cell dynamics. For this, we used a FRET sensor which shows distributions of phosphorylated RhoA in space and time (Fig. $4 \mathrm{a}$ and Ref. ${ }^{18,29}$, see Methods and Fig. S4a-C). In MDCK rings, we found the largest activity at inner and outer boundaries, where acto-myosin cables are assembled (Movie S9, Fig. 4b and Fig. 3a). This supports the contractile nature of these fibers. Accordingly, we found that the closure time of rings is shorter for large FRET activities in cables (see Fig. S4d-e). We can conclude that regulation by RhoA is tightly correlated with spatial evolution of rings.

Building on this result, we sought to test whether correlation between RhoA and cell velocity could be at play in the onset of coherence. We analyzed locally and globally velocity fields and their associated FRET maps (Fig. 4c and Fig. S4f-g, see also Methods). We found that high velocity regions for cells were correlated with low FRET levels (Fig. 4d,e). This suggests an inverse relationship between the two quantities. Cells move faster with lower levels of FRET. Accordingly, we next looked for potential links between the onset of coherence and FRET levels, assuming that lower FRET may facilitate motion. We saw that rings with higher FRET at initial time $t_{0}$ could not reach coherence (Fig. 4f). This further supports the notion that RhoA activity is essentially integrated in cell velocity. We then turned to numerical simulations to check whether the onset of collective motions could happen with basic interactions rules. 


\section{Numerical simulations reproduce the ring dynamics qualitatively and quantitatively}

Since we tracked cellular velocity and polarity in experiments, we selected a Vicsektype model ${ }^{30}$ to compare theoretical predictions with cellular readouts. We extended the model by the incorporation of free elastic boundaries to capture the potential contributions of acto-myosin cables. With basic rules of interactions between neighbouring cells for velocity and polarity and between cells and their boundaries, we performed 'numerical experiments' while keeping the same physical parameters measured mainly in our study (see Fig. S5a-f and Supplementary Material).

We report in Fig. 5 the comparisons in shapes and coherence values across conditions. The ring phenotypes are reproduced with multicellular fingers or clusters for distinct colony diameters (Fig. 5a and Movie S10-13). Similarly, the coherence index is quantitatively obtained with excellent precision (see Fig. $5 b$ ) together with its typical time dependence (Fig. 5c). Also, the tug-of-war between polarities correlated with the time required to reach coherence for simulated rings with quantitative consistency with experimental times (Fig. 5d). In addition, we could probe numerically the potential contribution of active forces at cables : interestingly, they were required for the onset of coherence (Fig. 5e). Finally, we tested the effect of having a cell with no cable within the ring, thereby modelling the caldesmon experiment (Fig. 3f) : the phenotype of this cell escape was reproduced as well (Fig. $5 f$ and Movie S14), substantiating the confinement role of cables. Altogether our numerical experiments based on measured cellular parameters and with basic interactions for single cells show that we can capture the main features for the onset of coherence with no need to incorporate parameters other than polarity, velocity, and active cables at boundaries.

\section{DISCUSSION}

We show that coherence of cellular movements emerges when the system size is below the coherence length inherent to MDCK cells as reported previously on epithelial disks ${ }^{20,21,31,32}$. Cells align their polarities within the ring and this step sets the onset of coherence. Furthermore, acto-myosin cables act as internally driven constrains confining cells tangentially. RhoA activity is larger at boundaries colocalizing with cables and is inversely related to the cell maximum velocity. Finally, with these ingredients, experimental and numerical rings share qualitative and quantitative features, supporting the relevance of our minimal framework for cell interactions. We show that active forces at cables are critical to orient cell polarity and contribute to coherence.

\section{Internally driven coherence versus externally driven coherence}

Emergence of coherent flows has been studied in vitro in confined situations ${ }^{20,21,25}$. Cells were chemically or mechanically confined and cannot escape their adhesive patterns ${ }^{20,21}$. This led to distinct phenomena - although with similar velocities of 20 $\mu \mathrm{m} . \mathrm{h}^{-1}$ - where the coherence is not driven only by the cellular characteristics but also 
by the boundaries of confinement as suggested by previous theoretical works ${ }^{33}$. Cells rotate mainly along the boundary driving a solid-like rotation. In Ref. ${ }^{25}$, this led to rotations even on rings with a diameter of $1 \mathrm{~mm}$, far above the natural coherence length of the epithelial cell line. In our case, we let cells spontaneously self-organize without confinement and we decreased the pattern size to initiate the onset of coherence. Remarkably, the symmetry of rotation clockwise-counterclockwise is broken on average, in contrast to former studies in confinement ${ }^{25}$ but in agreement with the bias reported in other studies ${ }^{23,24,34}$ with potential roles for left-right asymmetry during development.

\section{RhoA activity and its inverse relation with velocity}

We report for the first time to our knowledge live activity of RhoA integrated over an entire multicellular system. We considered MDCK rings as a continuum material where RhoA has its own dynamics and extracted FRET levels through time and space. Our results point to a simplification in their connection : RhoA and velocity are inversely related, which allows to consider only velocities for the model. This simplification would need to be further tested in other situations but it opens an interesting scheme for a simple relation between RhoA activity and cell velocity.

\section{Implication of coherent motions in vivo}

Our velocities found during coherent motion were close to the ones found during Drosophila egg chamber development ${ }^{10,11}$. In this context, follicle cells collectively rotate along the chamber and the presence of lamellipodia is reported ${ }^{10}$. This could potentially mean that the phenomena described in this work on $2 \mathrm{D}$ rings, driven by cell "cryptic" lamellipodia, act also during these in vivo processes. Also, coherent rotations have been reported in various morphogenetic events in vivo in mammary acini, in egg chamber and in testis rotation in Drosophila ${ }^{10-13,35}$. We propose that these phenomena could be generic and experienced by any growing tissues as suggested also by a recent theoretical study ${ }^{36}$. If right, spontaneous rotation could occur when coherence lengths of each epithelial layer would be similar to dimensions of tissues. It would be interesting to compare coherence lengths across model systems to evaluate whether this generic property of living matter is physiological and is important for optimal development.

\section{Acknowledgments}

We thank J. Van Unen and M. Inamdar for discussions and feedbacks. We also thank A. Honigmann for kindly sharing the ZO1-GFP MDCK cell line, the Imaging Platform of IGBMC, and the Riveline Lab. for help and discussions. S.L.V. is supported by the University of Strasbourg and by la Fondation pour la Recherche Médicale. D.R., M.S. and L.N. acknowledge supports from Idex Unistra and from the Cell Physics Master at the University of Strasbourg. O.P. and D.R. thank funding from SNF Sinergia. This study has been also supported by a French state fund through the Agence Nationale de la Recherche under the frame program Investissements d'avenir labeled ANR-10-IDEX-0002-02. D.R. and O.P. acknowledge funding from a Swiss National Science Fundation Sinergia grant 


\section{REFERENCES}

1. Munjal, A., Philippe, J., Munro, E. \& Lecuit, T. A self-organized biomechanical network drives shape changes during tissue morphogenesis. (2015) doi:10.1038/nature14603.

2. Nishikawa, M., Naganathan, S. R., Jülicher, F. \& Grill, S. W. Controlling contractile instabilities in the actomyosin cortex. Elife 1-21 (2017) doi:10.7554/eLife.19595.

3. Sit, S. T. \& Manser, E. Rho GTPases and their role in organizing the actin cytoskeleton. J. Cell Sci. 124, 679-683 (2011).

4. Ridley, A. J. \& Hall, A. The small GTP-binding protein rho regulates the assembly of focal adhesions and actin stress fibers in response to growth factors. Cell 70, 389-399 (1992).

5. Comelles, J. et al. Elongations of epithelial colony in vitro $\square$ : symmetry breaking through collective effects. bioRxiv 1-15 (2019).

6. Yam, P. T. et al. Actin-myosin network reorganization breaks symmetry at the cell rear to spontaneously initiate polarized cell motility. J. Cell Biol. 178, 12071221 (2007).

7. Arboleda-Estudillo, Y. et al. Movement Directionality in Collective Migration of Germ Layer Progenitors. Curr. Biol. 20, 161-169 (2010).

8. Jain, A. et al. Regionalized tissue fluidization is required for epithelial gap closure during insect gastrulation. Nat. Commun. 11, (2020).

9. Münster, S. et al. Attachment of the blastoderm to the vitelline envelope affects gastrulation of insects. Nature 568, 395-399 (2019).

10. Cetera, M. et al. Epithelial rotation promotes the global alignment of contractile actin bundles during Drosophila egg chamber elongation. Nat. Commun. 5, (2014).

11. Haigo, S. L. \& Bilder, D. Global tissue revolutions in a morphogenetic movement controlling elongation. Science (80-. ). 331, 1071-1074 (2011).

12. Wang, H., Lacoche, S., Huang, L., Xue, B. \& Muthuswamy, S. K. Rotational motion during three-dimensional morphogenesis of mammary epithelial acini relates to laminin matrix assembly. Proc. Natl. Acad. Sci. U. S. A. 110, 163168 (2013).

13. Tanner, K., Mori, H., Mroue, R., Bruni-Cardoso, A. \& Bissell, M. J. Coherent angular motion in the establishment of multicellular architecture of glandular tissues. Proc. Natl. Acad. Sci. U. S. A. 109, 1973-1978 (2012).

14. Selmeczi, D., Mosler, S., Hagedorn, P. H., Larsen, N. B. \& Flyvbjerg, H. Cell Motility as Persistent Random Motion $\square$ : Theories from Experiments. Biophys. J. 89, 912-931 (2005).

15. Vedula, S. R. K. et al. Emerging modes of collective cell migration induced by geometrical constraints. Proc. Natl. Acad. Sci. U. S. A. 109, 12974-12979 (2012).

16. Ladoux, B. \& Mège, R. M. Mechanobiology of collective cell behaviours. Nat. Rev. Mol. Cell Biol. 18, 743-757 (2017).

17. Reffay, M. et al. Interplay of RhoA and mechanical forces in collective cell migration driven by leader cells. Nat. Cell Biol. 16, 217-223 (2014). 
18. Pertz, O., Hodgson, L., Klemke, R. L. \& Hahn, K. M. Spatiotemporal dynamics of RhoA activity in migrating cells. Nature 440, 1069-1072 (2006).

19. Turing, A. M. The chemical basis of morphogenesis. Philos. Trans. R. Soc. London 237, 37-72 (1952).

20. Doxzen, K. et al. Guidance of collective cell migration by substrate geometry. Integr. Biol. (United Kingdom) 5, 1026-1035 (2013).

21. Deforet, M., Hakim, V., Yevick, H. G., Duclos, G. \& Silberzan, P. Emergence of collective modes and tri-dimensional structures from epithelial confinement. Nat. Commun. 1-9 (2014) doi:10.1038/ncomms4747.

22. Petitjean, L. et al. Velocity fields in a collectively migrating epithelium. Biophys. J. 98, 1790-1800 (2010).

23. Chin, A. S. et al. Epithelial cell chirality revealed by three-dimensional spontaneous rotation. Proc. Natl. Acad. Sci. U. S. A. 115, 12188-12193 (2018).

24. Tee, Y. H. et al. Cellular chirality arising from the self-organization of the actin cytoskeleton. Nat. Cell Biol. 17, 445-457 (2015).

25. Jain, S. et al. The role of single-cell mechanical behaviour and polarity in driving collective cell migration. Nat. Phys. 16, 802-809 (2020).

26. Nier, V. et al. Tissue fusion over nonadhering surfaces. Proc. Natl. Acad. Sci. U. S. A. 112, 9546-9551 (2015).

27. Mayer, M., Depken, M., Bois, J. S., Jülicher, F. \& Grill, S. W. Anisotropies in cortical tension reveal the physical basis of polarizing cortical flows. Nature 467, 617-621 (2010).

28. Helfman, D. M. et al. Caldesmon inhibits nonmuscle cell contractility and interferes with the formation of focal adhesions. Mol. Biol. Cell 10, 3097-3112 (1999).

29. MacHacek, M. et al. Coordination of Rho GTPase activities during cell protrusion. Nature 461, 99-103 (2009).

30. Vicsek, T., Czirk, A., Ben-Jacob, E., Cohen, I. \& Shochet, O. Novel type of phase transition in a system of self-driven particles. Phys. Rev. Lett. 75, 12261229 (1995).

31. Segerer, F. J., Thüroff, F., Piera Alberola, A., Frey, E. \& Rädler, J. O. Emergence and persistence of collective cell migration on small circular micropatterns. Phys. Rev. Lett. 114, 1-5 (2015).

32. Zorn, M. L., Marel, A. K., Segerer, F. J. \& Rädler, J. O. Phenomenological approaches to collective behavior in epithelial cell migration. Biochim. Biophys. Acta - Mol. Cell Res. 1853, 3143-3152 (2015).

33. Soumya, S. S. et al. Coherent Motion of Monolayer Sheets under Confinement and Its Pathological Implications. PLoS Comput. Biol. 11, 1-30 (2015).

34. Naganathan, S. R. a., Fürthauer, S., Nishikawa, M., Jülicher, F. \& Grill, S. W. Active torque generation by the actomyosin cell cortex drives left-right symmetry breaking. Elife 3, e04165 (2014).

35. Chougule, A. et al. The Drosophila actin nucleator DAAM is essential for leftright asymmetry. PLoS Genet. 16, 1-23 (2020).

36. Maitra, A. \& Lenz, M. Spontaneous rotation can stabilise ordered chiral active fluids. Nat. Commun. 10, 1-6 (2019).

37. Beutel, O., Maraspini, R., Pombo-García, K., Martin-Lemaitre, C. \& Honigmann, A. Phase Separation of Zonula Occludens Proteins Drives Formation of Tight Junctions. Cell 179, 923-936.e11 (2019).

38. Etournay, R. et al. TissueMiner: A multiscale analysis toolkit to quantify how cellular processes create tissue dynamics. Elife 5, 1-28 (2016). 


\section{MATERIAL AND METHODS}

\section{Micro-contact printing}

Ring motifs are patterned on glass coverslips using micro-contact printing. Polydimethylsiloxane (PDMS; 1:10 w/w cross-linker:pre-polymer) (Sylgard 184 kit, Dow Corning, cat. DC184-1.1). Stamps are produced with UV-photolithography. Stamps are rinsed with $70 \%$ ethanol and then rendered hydrophilic by exposure to oxygen plasma (30s) (Diener Electronic, cat. ZeptoB). They are next incubated with a $10 \mu \mathrm{g} / \mathrm{mL}$ rhodamine-labelled fibronectin solution (Cytoskeleton, cat. FNR01-A) for $1 \mathrm{~h}$ and then dried at room temperature for about $5 \mathrm{~min}$. In the meantime, glass coverslips are functionalized by vapour phase for $1 \mathrm{~h}$ with 3-(mercapto)propyltrimethoxysilane (FluroChem). After incubation of both the coverslip and PDMS, the stamp is deposited onto the glass slide and kept in contact during 30min. To ensure a proper transfer between the two surfaces, a $50 \mathrm{~g}$ weight is placed on the top of the stamp. Finally, the stamp is removed and the printed coverslip is washed with PBS $1 \mathrm{X}$ and stored in Milli-Q water. Non-printed areas are passivated with $0.1 \mathrm{mg} / \mathrm{mL}$ PLL-g-PEG (in 1mM HEPES pH 7.4, SuSoS AG, cat. SZ33-15) for $20 \mathrm{~min}$ at room temperature.

\section{Cell Culture}

MDCK cells stably transfected with E-cadherin-DsRED are cultured in low glucose Dulbecco's Modified Eagle's (DMEM) with 10\% Foetal Bovine Serum (FBS) and 1\% Penicillin-Streptomycin. Cells are kept subconfluent (at $\sim 70 \%$ ). Prior experiments, cells are detached with Trypsin-0.25\% EDTA (Fisher Scientific, cat. 11570626) and centrifuged at 500rpm during $3 \mathrm{~min}$. The pellet is then re-suspended in DMEM $1 \%$ FBS. This low serum concentration will further prevent attachment on the passivated surface during seeding. After $1 \mathrm{~h}$ incubation, the printed surface is carefully washed out to remove cells from the non-printed area. After washout, DMEM is replaced by L-15 Leibovitz Medium (Fisher Scientific, cat. 11540556) supplemented with 1\% FBS and $1 \%$ Penicillin-Streptomycin and the sample is incubated $1 \mathrm{~h}$ under the microscope at $37^{\circ} \mathrm{C}$ before acquisition. This last incubation allows cells to spread on patterns and ensures that rings are closed at the onset of the experiment.

For laser ablation experiments, MDCK stably transfected with GFP-Myosin Regulatory Light Chain (MRLC) were used. Tight junctions were imaged with a MDCK cell line genetically engineered with CRISPR-Cas9 to express ZO1-GFP ${ }^{37}$. We generated a MDCK cell line stably expressing the RhoA FRET biosensor using Piggy-Bac engineering. Briefly, cells were transfected with the biosensor plasmid together with a transposase. After integration into the genome, fluorescent cells were sorted with flow cytometry and kept under antibiotics pressure for 3 weeks.

\section{Transfection}

Plasmids for mosaic experiments such as Actin-GFP or Caldesmon-GFP were incubated with cells using Lipofectamin (Invitrogen, cat. 11668030). One day prior 
experiment, cells at 50\% confluency in a 6-well plate were incubated for 4-6h with $1 \mu \mathrm{g}$ of DNA and $10 \mu \mathrm{L}$ of lipofectamin mixed in Opti-MEM medium. After incubation, wells are washed out and the medium is replaced by fresh DMEM 10\% FBS.

\section{Standard time-lapse microscopy}

Phase contrast images were acquired through a $10 \mathrm{x}$ objective (NA $=0.25)$ with a frame rate of 1 image every $10 \mathrm{~min}$. Patterns were systematically checked prior the time-lapse start with a standard epifluorescence lamp (FluoArc Hg Lamp) coupled with a rhodamine fluorescence filter. The very same set-up was used to acquire cells during mosaic experiments (actin-GFP and caldesmon-GFP). Tight junctions (ZO1GFP) were imaged with a confocal microscope (Leica SP8 inverted) and an autofocus system. All z-planes were taken and finally projected onto one single plane.

\section{Biosensor FRET activity}

Images were acquired with a confocal microscope (Leica SP8 inverted) with the pinhole opened to the maximum in order to increase slice thickness and the signal to noise ratio for the analysis. We used two Photo Multiplier Tubes (PMTs) as detectors. Bandwidths were chosen as follow: 472nm-512nm for mTFP1 (CFP analog, donor) and $523 \mathrm{~nm}-544 \mathrm{~nm}$ for Venus (YFP analog, acceptor). mTFP1 was excited by an argon laser at $468 \mathrm{~nm}$ with $15 \%$ of power and emission for mTFP1 (donor) and Venus (FRET) were simultaneously acquired by the two PMTs. Images were then corrected and processed with Biosensor Package from Danuser Lab (available on the following link: https://github.com/DanuserLab/Biosensor). Because it is a unimolecular biosensor, no bleedthrough correction was done. Prior analysis, histograms were manually resliced in order to remove high and close-to-zero boundary artefacts.

To check whether the FRET sensor was reliable, we performed additional tests: incubation with $1.0 \mu \mathrm{g} / \mathrm{ml} \mathrm{C3-transferase} \mathrm{(RhoA} \mathrm{inhibitor,} \mathrm{Cytoskeleton,} \mathrm{cat.} \mathrm{CT04)}$ and with $1.0 \mu \mathrm{g} / \mathrm{ml}$ of RhoA activator (Cytoskeleton, cat. CN03). Drugs led to decrease and increase of activities respectively (Fig. S4a-b). We also used a mutant sensor - F39A, unable to interact with the RBD domain ${ }^{18}$ - with the same acquisition parameters detailed above. This was associated with a significant decrease in the FRET signal (Fig. S4c).

\section{Laser ablation}

Laser ablations on acto-myosin cables were done using the FRAP module from a Leica SP-5 inverted microscope. The sample was imaged and ablated through a 40x oil objective (NA = 1.25) and an 800nm infrared laser ( $80 \mathrm{MHz}$, pulsed).

\section{Velocity fields and coherence length}

Velocity fields were generated using PIVlab 1.4 and performing cross-correlations. Coherence lengths $\xi$ were extracted from velocity fields with a custom-made Matlab code by computing the spatial correlation function of the tangential velocity $v_{\theta}$ : 


$$
C_{v_{\theta}}(\delta x, t)=\frac{\left\langle\overrightarrow{v_{\theta}}(\vec{x}+\delta \vec{x}, t) \cdot \overrightarrow{v_{\theta}}(\vec{x}, t)\right\rangle}{\left\langle v_{\theta}(\vec{x}, t)^{2}\right\rangle}
$$

Exponential decays are generated from the computations of $C_{v_{\theta}}$ and the intersections between the tangents at origin and $\mathrm{x}$-axis give $\xi$. Because $C_{v_{\theta}}$ was not always converging to 0 , we made linear fits on the 3 first points of $C_{v_{\theta}}$ in order to extract the tangent at origin.

To extract the maximal coherence length $\xi_{\max }$ presented in Figure 1c and Fig. S1a, velocity fields were averaged over intervals of 10 frames (100min) and the time interval leading to the largest correlation function was considered.

\section{Image processing}

Nuclei in RhoA ratiometric maps were removed using llastik and phase contrast images were processed with Fiji.

Since many factors could contribute to define physical polarity, we decided to track ZO1 marked cells - not in their anisotropy of signal - but for the well defined shapes. We confirmed that this polarity was consistent with the polarity defined by lamellipodia in the large majority of cases (Fig. S2a-b). Segmentation and extraction of single cell parameters in ZO1 experiments were done using Tissue Analyzer ${ }^{38}$ in Fiji.

\section{Model}

We designed a mechanistic-based minimal "Vicsek-type model" in which cells are treated as particles with polarity $\mathbf{p}$ and velocity $\mathbf{v}$, initially placed on a ring with two layers of cells matching the cell density and number tested in experiments. Their interaction rules are determined by alignment of polarities and by polarity relaxation to the direction of motion and by random fluctuations of polarities (see Annex for details). Velocities are also projected on the directions of polarities to the limit of volume exclusion. The inner and outer supra-cellular acto-myosin cables are modeled as segments connecting boundary cells with two physical elastic properties, stretching and bending energies. In addition to the force exerted by the cables on the cells and its velocities, cables also act on cell polarity. The values of parameters are extracted from measurements and from estimates based on references (see Annex and Table I).

We show (see Fig. 5e) that with this passive treatment of cables and actual experimental values, we do not recapitulate coherent motion. Based on the Rho activity at both cables supporting an active stress generation, we add in the model an active cable force with estimates of the associated experimental values. Simulations based on the theoretical model reveal that cells undergo coherent motion for small rings. Moreover, when we increase the ring diameter keeping the same values for parameters, we reproduce the experimental dynamics with no further adjustments. These results support the relevance of our model. 


\section{FIGURES AND MOVIE CAPTIONS}

a.
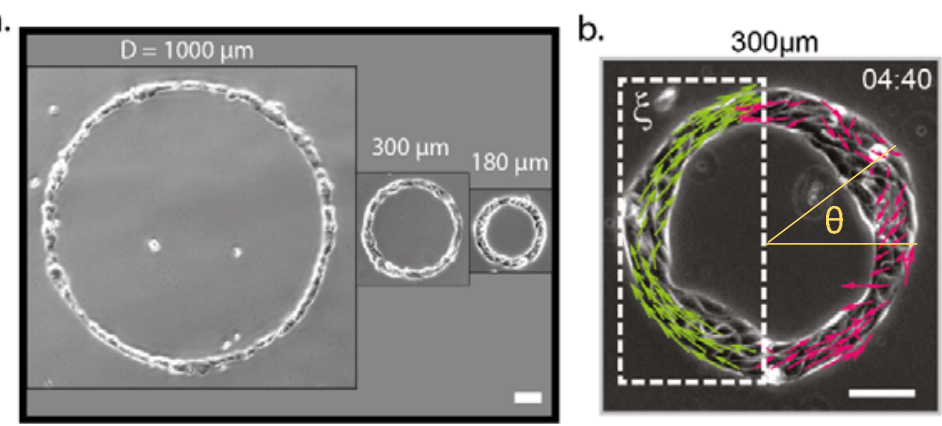

d.

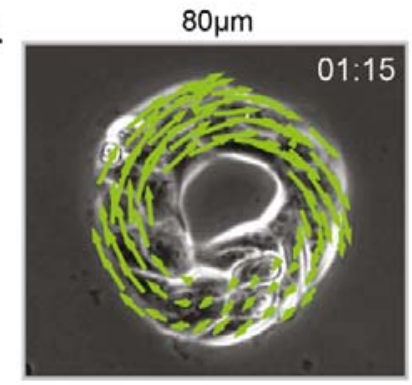

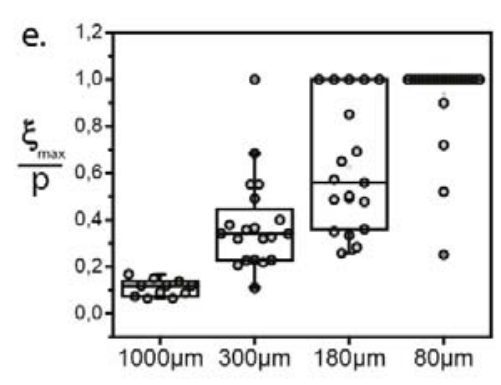

c.
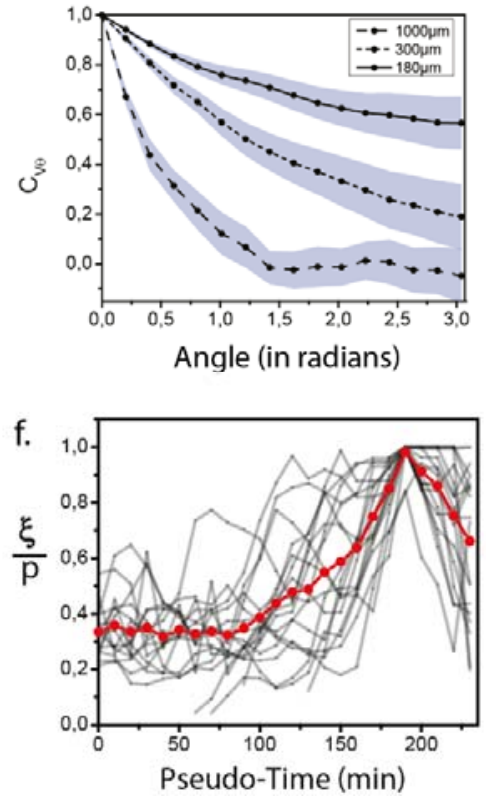

Figure 1. Acquisition of coherent motion. (a) MDCK rings of various diameters at intial time $t_{0}$. (b) Coherent flows of length $\xi$ can be extracted from the velocity fields. (c) Correlation functions of tangential velocity $v_{\theta}$. Sale bar $=50 \mu \mathrm{m}$. (d) $80 \mu \mathrm{m}$ ring with a perimeter below the coherence length undergoes spontaneous rotation. (e) $80 \mu \mathrm{m}$ rings are "rectified" and the majority of rings are coherent. $n_{1000}=11 ; n_{300}=20 ; n_{180}$ $=20 ; n_{80}=24$. (f) Temporal sequences are aligned on the coherence acquisition (- $\quad$ ) along a new pseudo-time. Coherence is a non-linear process taking place within $\sim 1 \mathrm{~h} 30$. 

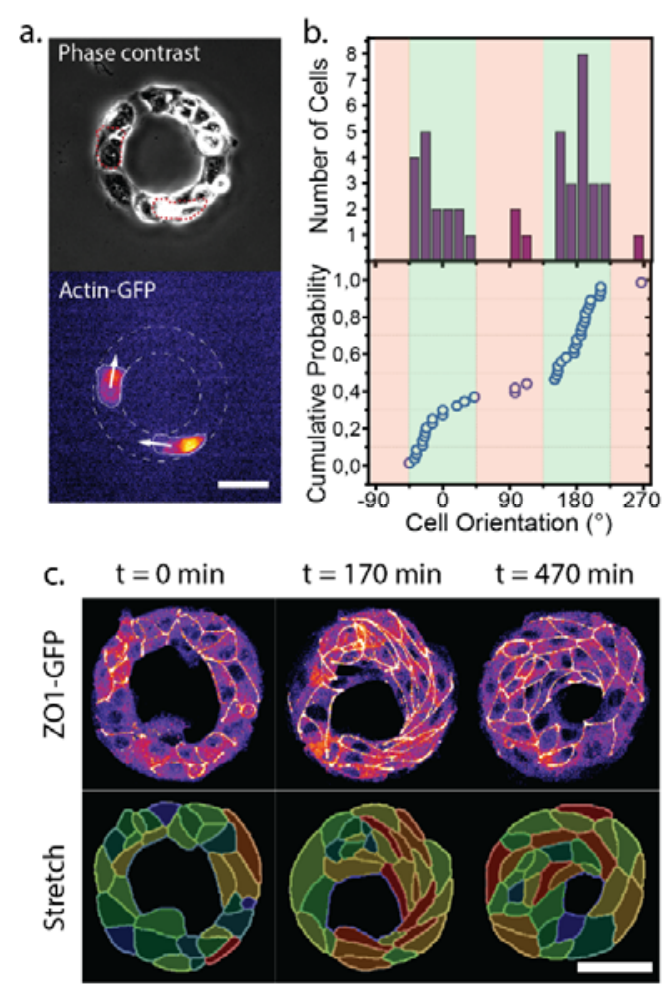

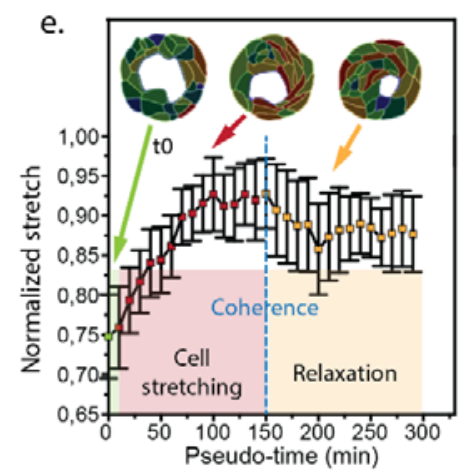

d.

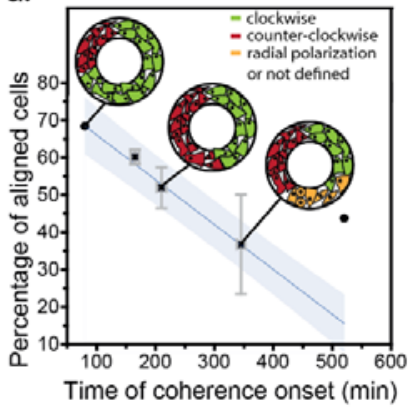

f.

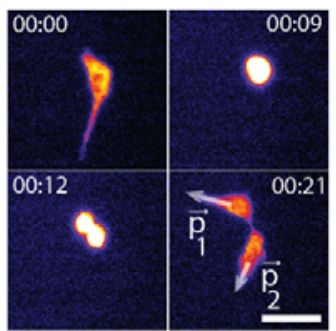

g.

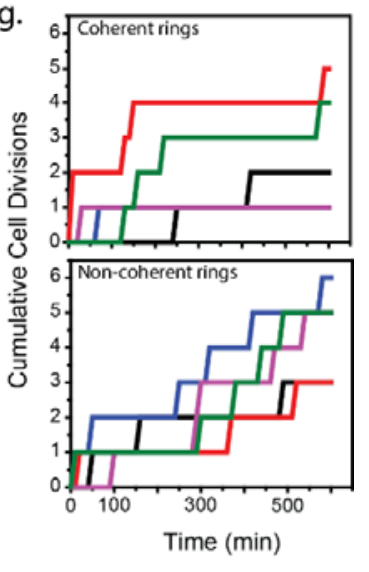

Figure 2. Cell polarity and density determine dynamics of coherent motion. (a) $80 \mu \mathrm{m}$ "mosaic" ring. Arrows indicate cell polarity based on the direction/orientation of cell lamellipodium. Scale bar $=50 \mu \mathrm{m}$. (b) Distribution of cell initial orientations within the rings, $\mathrm{n}_{\text {rings }}=12, \mathrm{n}_{\text {cells }}=42$. (c) Segmentation of multicellular rings based on $\mathrm{ZO} 1$ distributions. Single cell parameters such as cell elongation (stretch) can be extracted, red and blue largest and lowest stretch respectively. Scale bar $=50 \mu \mathrm{m}$. (d) Percentage of aligned cells based on the polarity given by ZO1 distributions. Data are binned with respect to the time of coherence onset. Data are represented as Mean \pm SD. $\mathrm{N}=10$. (e) Average normalized stretch (initial/max) aligned along a new pseudotime. The dashed blue line corresponds to coherence acquisition. Data are represented as Mean $\pm S D$. $N=10$. (f) Division of an actin-GFP labeled cell within a $80 \mu \mathrm{m}$ ring. Cell division generates daughter cells with opposite polarities $\mathbf{p}_{\mathbf{1}}$ and $\mathbf{p}_{\mathbf{2}}$. Scale bar $=20 \mu \mathrm{m}$. (g) Cumulative cell divisions as a function of time. Coherent rings exhibit bursts of divisions with large periods without divisions whereas non-coherent rings undergo regular divisions throughout the course of the experiment. For clarity, only 5 representative rings are shown for each case. Full population is available in Fig. S2. 
a.

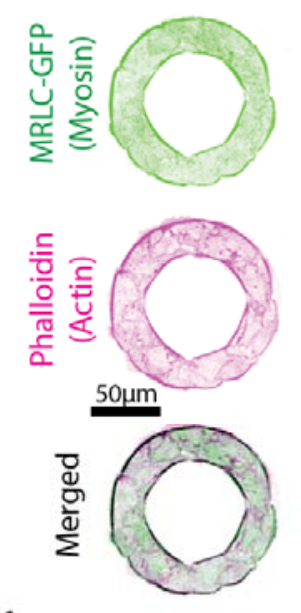

f.

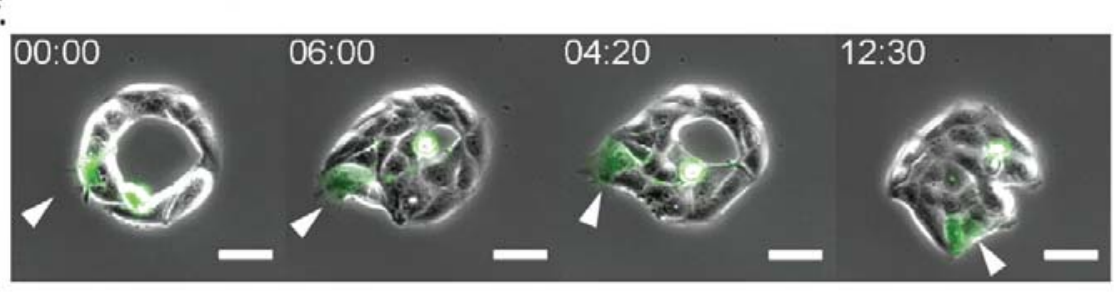

c.

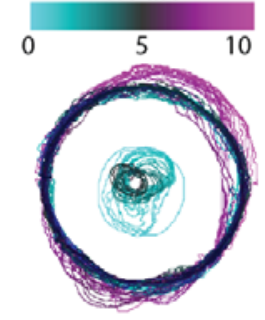

e.

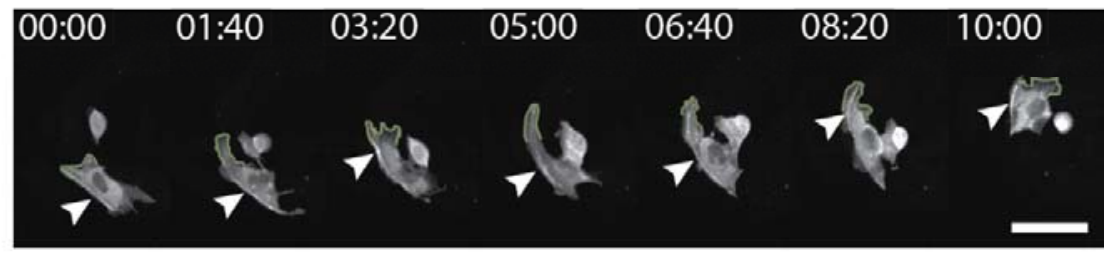

g.

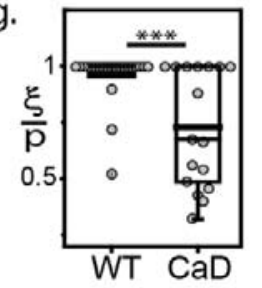

Figure 3. Acto-myosin cables as internally-driven constraints. (a) Immunostaining of a $80 \mu \mathrm{m}$ ring. Myosin and actin are visualized. (b) Tracking of the ring boundaries. The color code indicates time. The ring first closes inwards before extending outwards. (c) Kymographs showing acto-myosin ring retraction after laser ablation and cell-cell junction opening (as a control) after ablation. (d) Retraction velocity as a function of time after laser ablation. Exponential fits give estimates of $v_{0}$ defining the relative tension stored in the ring. (e) Actin vizualisation (lifeAct) of a transfected cell within a $80 \mu \mathrm{m}$ ring. The acto-myosin cable is self-generated by the cell during motion and constrains the polarity in a tangential orientation. White arrow indicates the cable, green contour underlines the lamellipodium boundary. Scale bar $=20 \mu \mathrm{m}$. (f) Time-lapse showing a CaD transfected cell (white arrow) escaping the multicellular ring and challenging coherence. Scale bar $=50 \mu \mathrm{m}$. (g) Coherence acquisition for non-transfected ring and rings with $\mathrm{CaD}$ transfected cells. There is a significant decrease in coherence $\left(p=7.10^{-4}\right)$ in the CaD rings. 
a.

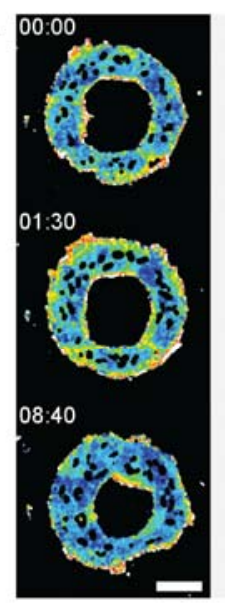

d.

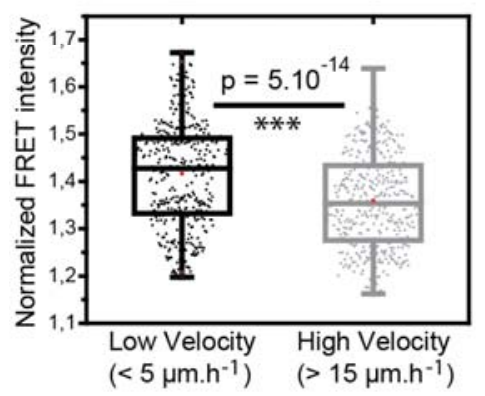

b.
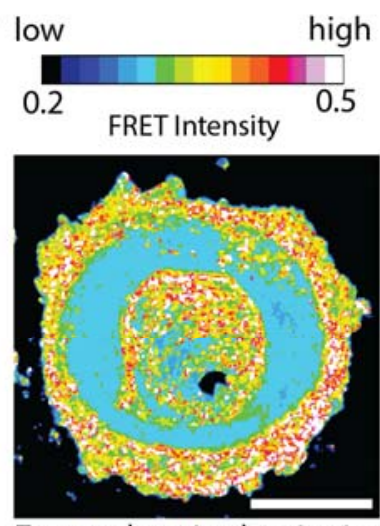

Temporal maximal projection

e.

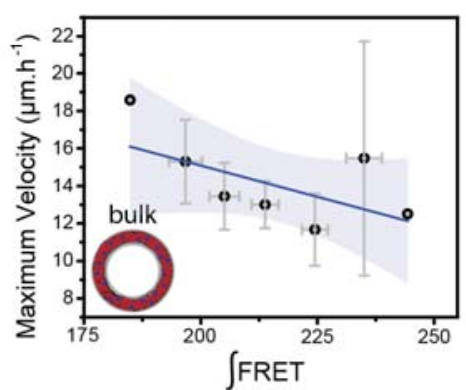

C.

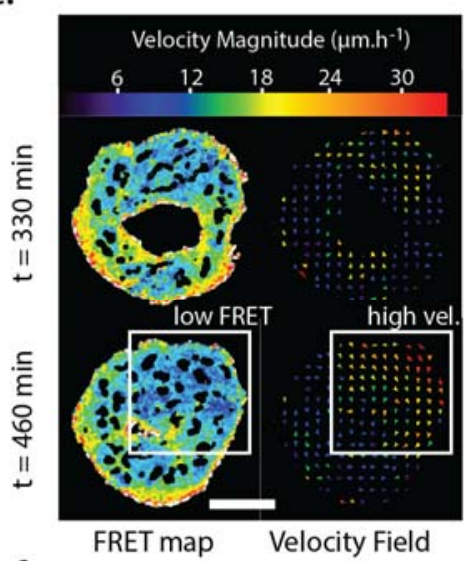

f.

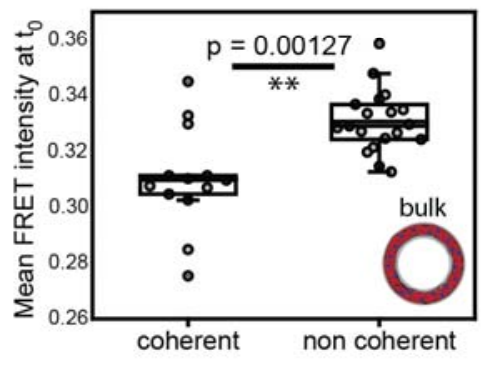

Figure 4. RhoA activity correlates with different ring behaviors. (a) RhoA activity on a $80 \mu \mathrm{m}$ ring as described by the FRET ratiometric map (left) associated to a velocity field (right). Scale bar $=50 \mu \mathrm{m}$. (b) Temporal maximal projection showing highest FRET signals at ring boundaries. Scale bar $=50 \mu \mathrm{m}$. (c) FRET map for each frame is represented with its associated velocity field. Scale bar $=50 \mu \mathrm{m}$. Comparison is shown in (d) where high FRET regions correlate with low velocity. (e) Maximum velocity of the ring (with both radial and tangential components) is represented as a function of the averaged FRET level integrated over the entire experiment. (f) FRET intensity at $t_{0}$ averaged over the cellular "bulk" excluding cables. Initial levels of RhoA activity are plotted for different coherent behaviors. High activity is correlated with low coherence. Data in (e) are binned; full distributions are shown in Fig. S4h. Same color codes for (a), (b) and (c) for FRET signals. 
a.
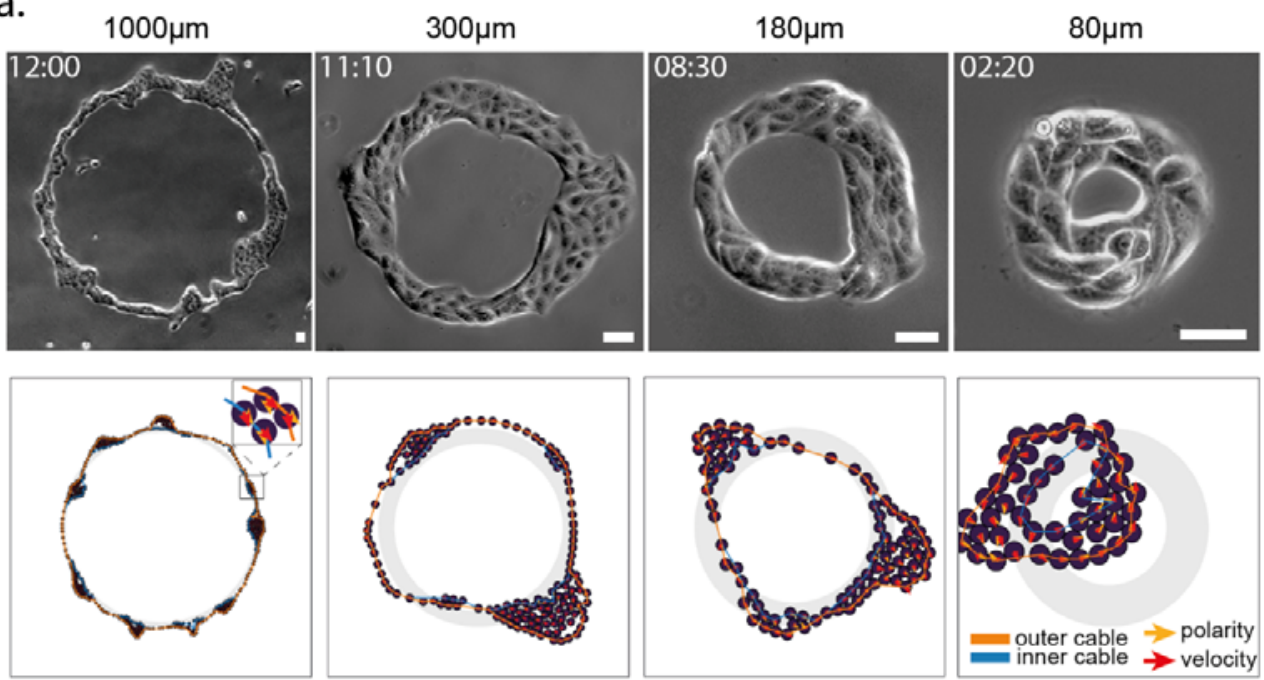

b.

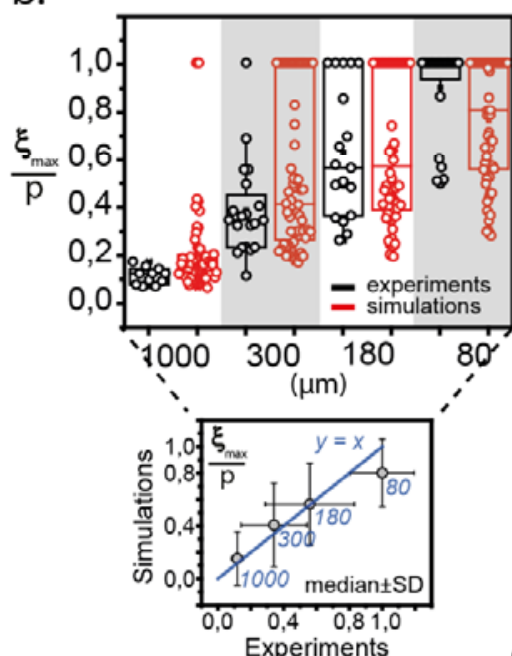

f.
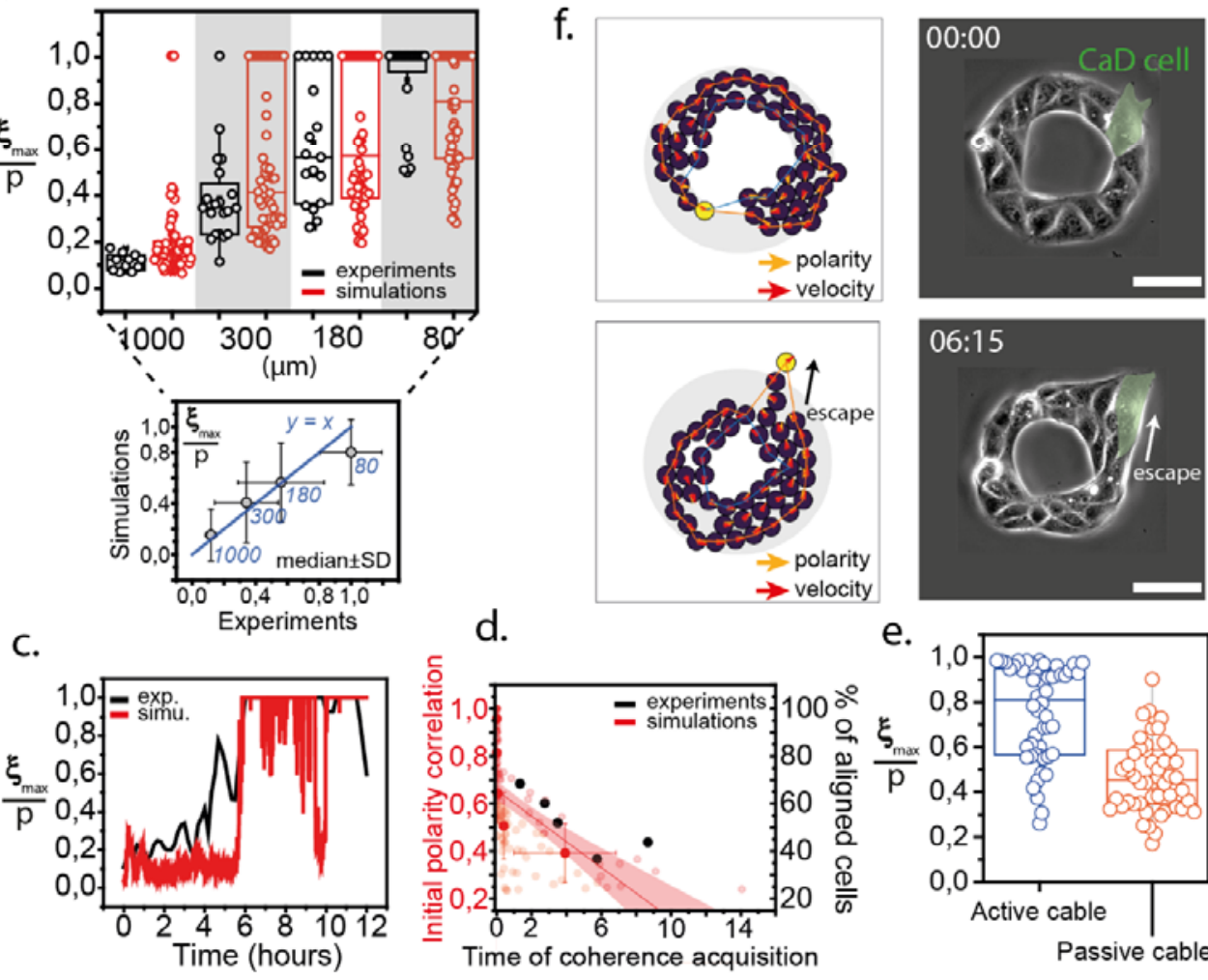

d.
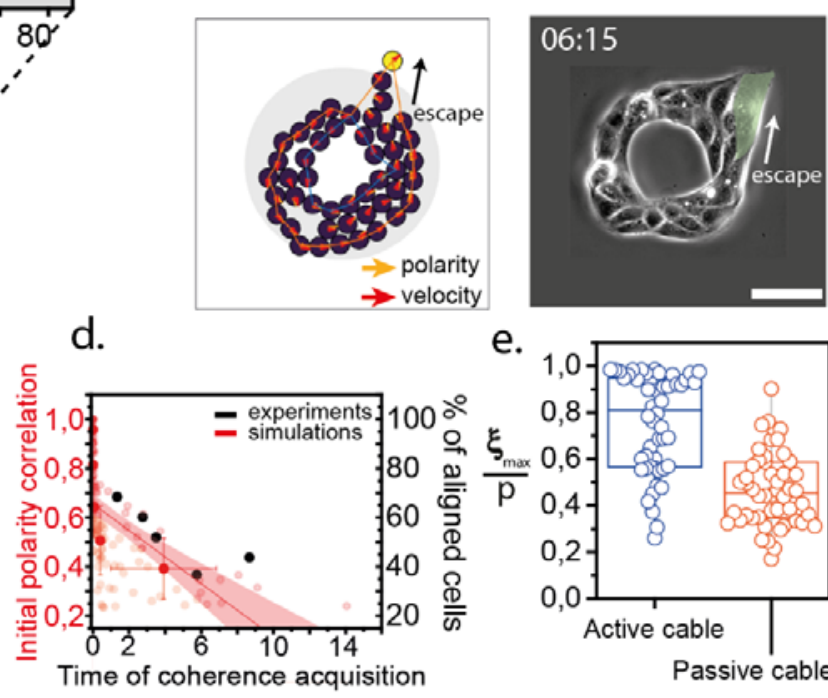

e.

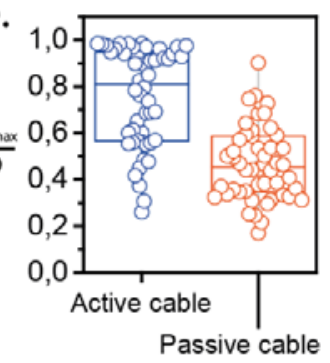

Figure 5. Theoretical modelling of multicellular rings. Experiments are compared with simulations. (a) Rings of different diameters are represented (top) when typical motifs appear, fingering for large diameters, clusters for intermediate rings, closure for smaller rings. Morphology is reproduced by numerical simulations (bottom). Scale bar $=50 \mu \mathrm{m}$. Time in hh:mm; simulations times (in hh:mm, from left to right): 05:24; $17: 56 ; 04: 00 ; 04: 57$. (b) Coherence index as a function of ring diameters for simulations and experiments, with an inset comparing median values. (c) Dynamics of coherence acquisition in silico compared with a typical MDCK ring. (d) Initial polarity correlation as a function of the time of coherence acquisition. The decay 
bioRxiv preprint doi: https://doi.org/10.1101/2021.11.11.468187; this version posted November 12,2021 . The copyright holder for this preprint (which was not certified by peer review) is the author/funder, who has granted bioRxiv a license to display the preprint in perpetuity. It is made available under aCC-BY-NC-ND 4.0 International license.

observed in experiments is similar to simulated rings. (e) Coherence index for two different populations of MDCK rings: activity within cable facilitates acquisition of coherence. (f) Caldesmon experiment and cellular 'escape' are reproduced with simulations. The yellow particle represents the $\mathrm{CaD}$ cell and its cable parameters are set to zero. Scale bar $=50 \mu \mathrm{m}$. Time in hh:mm. Simulation times (in hh:mm, from top to bottom): 05:20; 13:03. 


\section{SUPLLEMENTARY FIGURES}

a
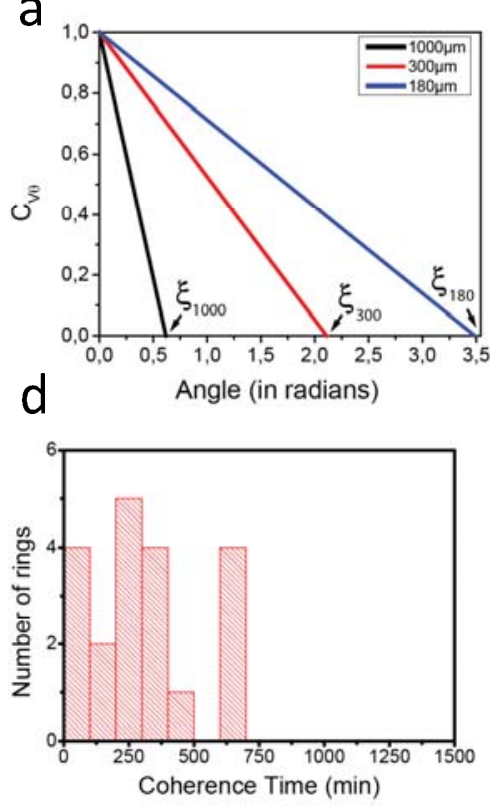

b

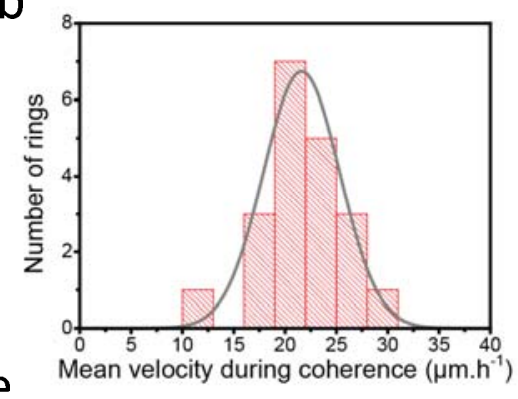

e

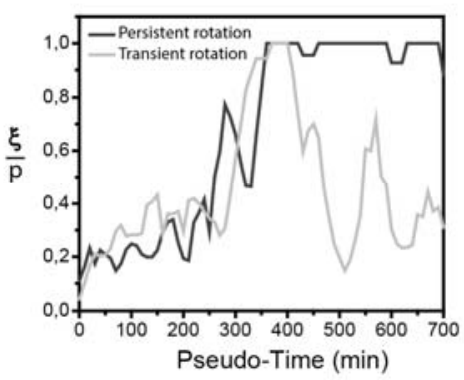

C

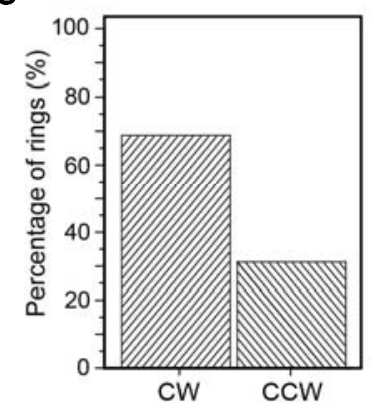

f

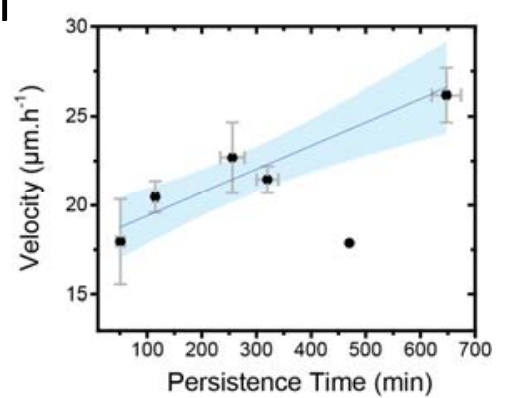

Figure S1. Different populations of coherent rings. (a) Tangents at origin from the correlation functions of the tangential velocity gives $\xi_{\max }$ for each configuration. (b) Distribution of ring velocities during coherence. (c) Clockwise (CW) orientation is favored during rotation compared to the counter-clockwise (CCW) orientation. (d) Distribution of coherence durations. (e) Two ring populations appear after the onset of coherence: (i) rings undergoing persistent rotation and (ii) rings exhibiting transient rotation with a fast decay of the coherence index. (f) Coupling between ring velocity and persistence of coherent motion. Data are binned with respect to time. Timers in hh:mm. Scale bars $=50 \mu \mathrm{m}$. 


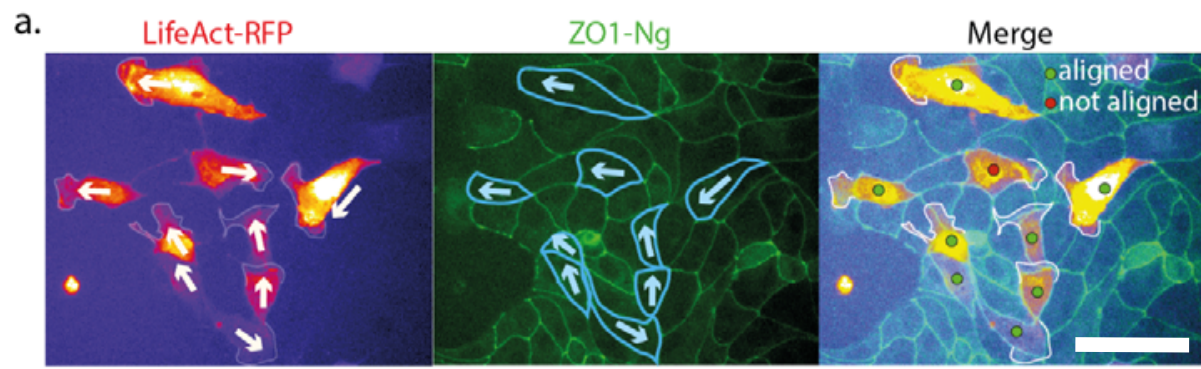

b.

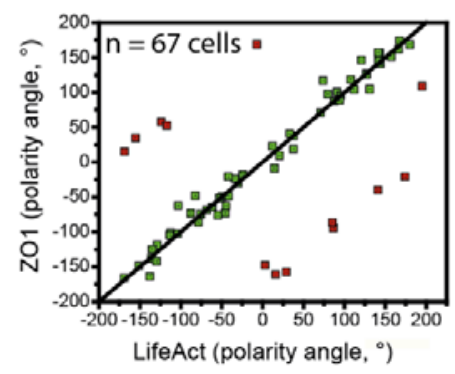

c.

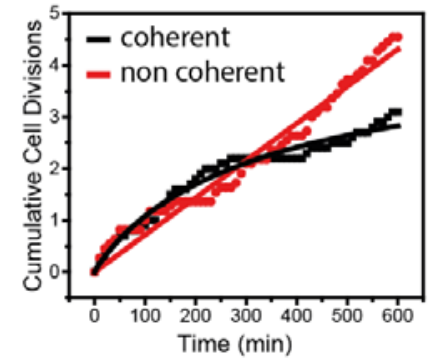

Figure S2. Extraction of single cell parameters within the ring. (a) Epithelium of MDCK cells expressing ZO1-Ng transfected with LifeAct-RFP. Actin visualization allows to identify lamellipodia and extract cell polarity. Scale bar $=20 \mu \mathrm{m}$. Cell polarity given by the lamellipodium and determined by ZO1 distribution are compared in (b). Green $=$ correlated, red $=$ non-correlated. (c) Complete distributions of cumulative cell divisions shown in Fig. 2.

a

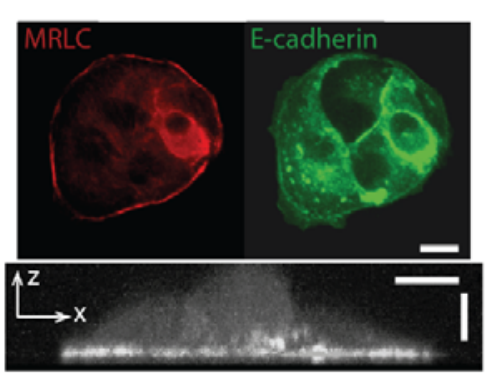

b

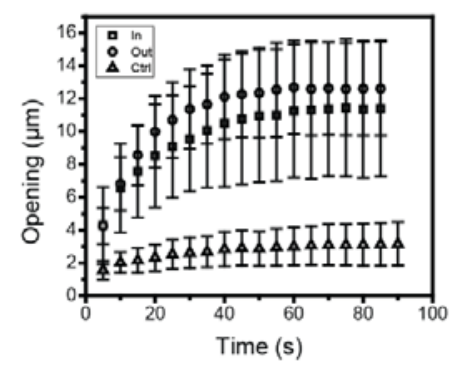

C

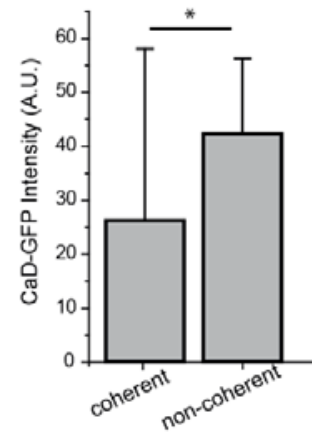

Figure S3. Acto-myosin cables assembly and dynamics. (a) Acto-myosin cable at the boundary of WT MDCK colony on a glass surface. Scale bars $=5 \mu \mathrm{m}$. (b) Opening dynamics with laser ablation of acto-myosin cables and cell-cell junction (control-ctrl). (c) Fluorescence intensity of $\mathrm{CaD}$ transfected cells within the ring for coherent and non-coherent rings. Coherent rings correspond to lower $\mathrm{CaD}$ expressions. 

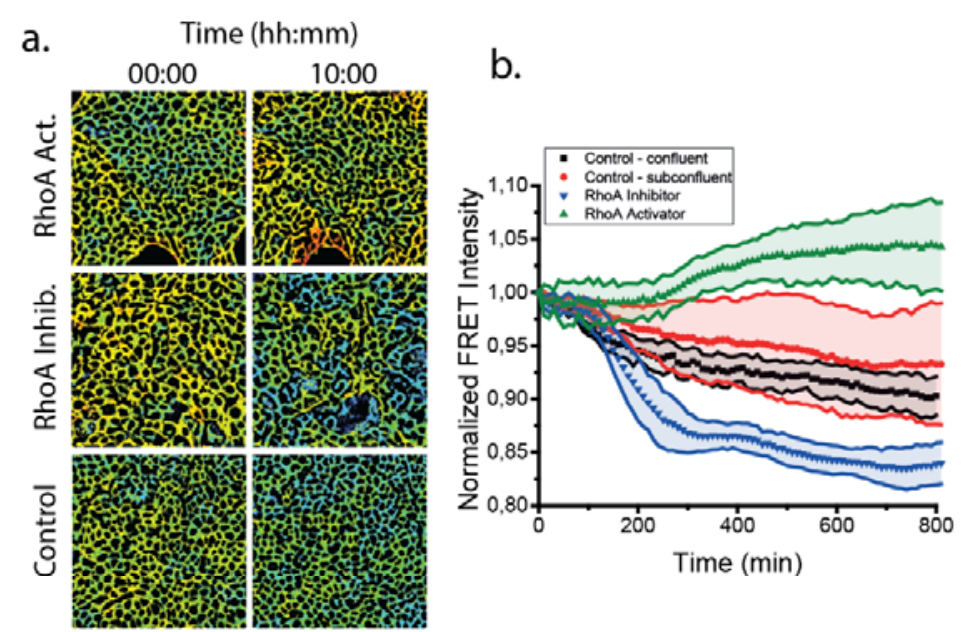

d.

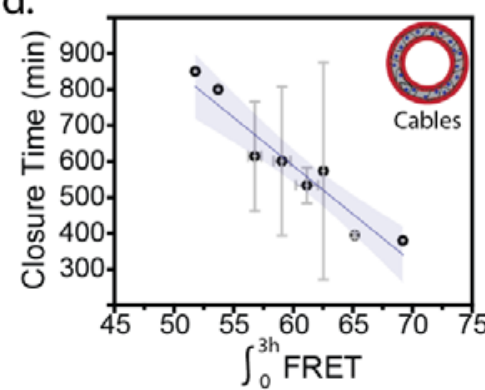

e.

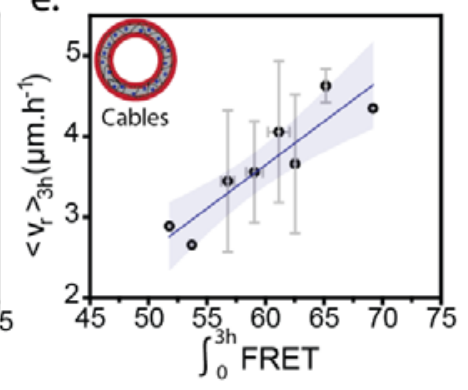

C.

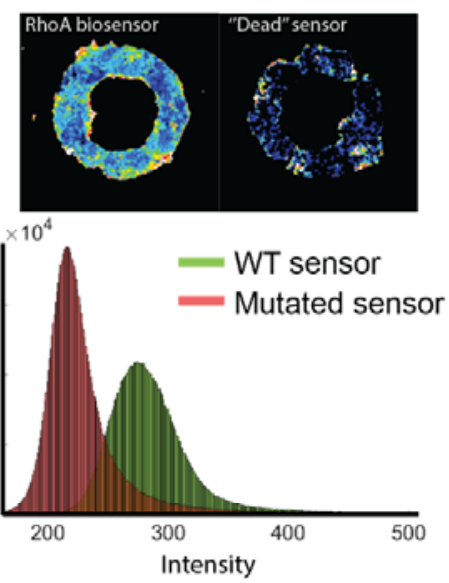

h.

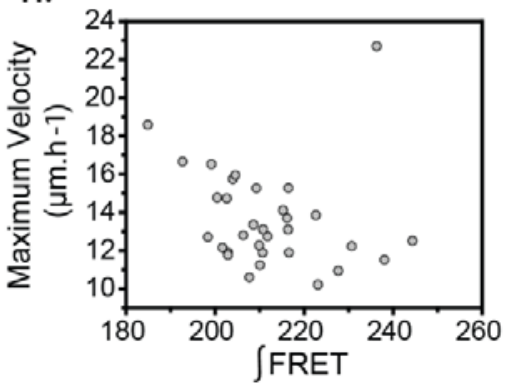

f.

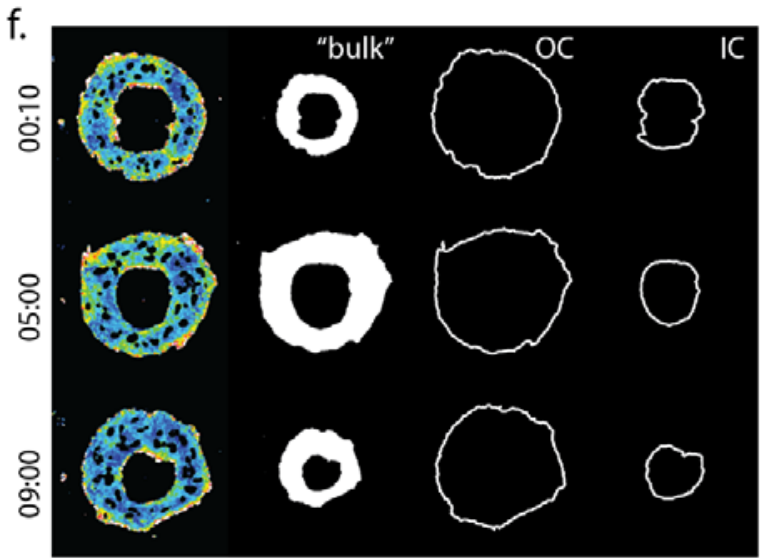

g.

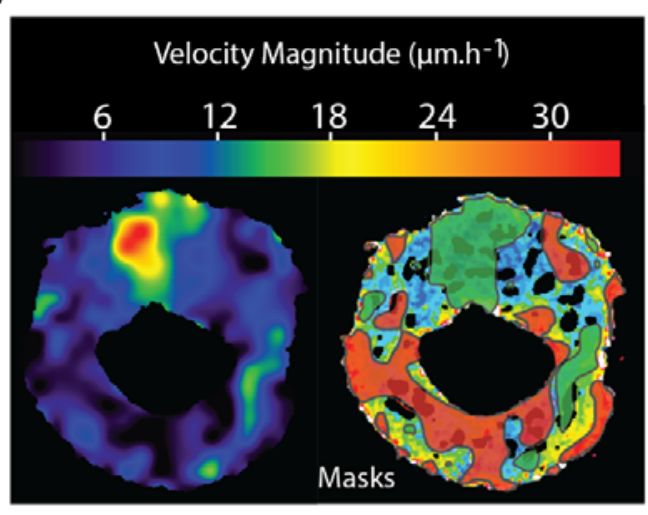

Figure S4. RhoA activity in rings. (a) MDCK epithelia treated with different RhoA regulating drugs together with the FRET signal quantification over time in (b). (c) FRET intensity acquired with a mutated sensor (described in ref ${ }^{18}$ ) shows a decrease in intensity. The set of experiments in panels (a-c) confirms the efficacy of our RhoA sensor and the proper images acquisition and analysis protocols. (d) Average FRET level at the cables, integrated over the initial time points (3h) correlated with the closure time. High RhoA activity at boundaries is associated with a faster ring closure. This read-out can be assessed by (c) where the average radial velocity is compared to the initial average FRET level. (f) Segmentation of the multicellular rings. Two regions are distinguished for analysis: the acto-myosin cables at the two interfaces and the cellular "bulk" within the two cables. (g) Velocity regions defined as low $\left(<5 \mu \mathrm{m} \cdot \mathrm{h}^{-1}\right)$ and high $\left(>15 \mu \mathrm{m} \cdot \mathrm{h}^{-1}\right)$ associated with their masked FRET 
map (green mask = high velocity, red mask = low velocity). (h) Full data of the FRET analysis shown in Fig. 4e.

a.

Presence of a lamellipodium (polarity readout)

No Migration

Migration

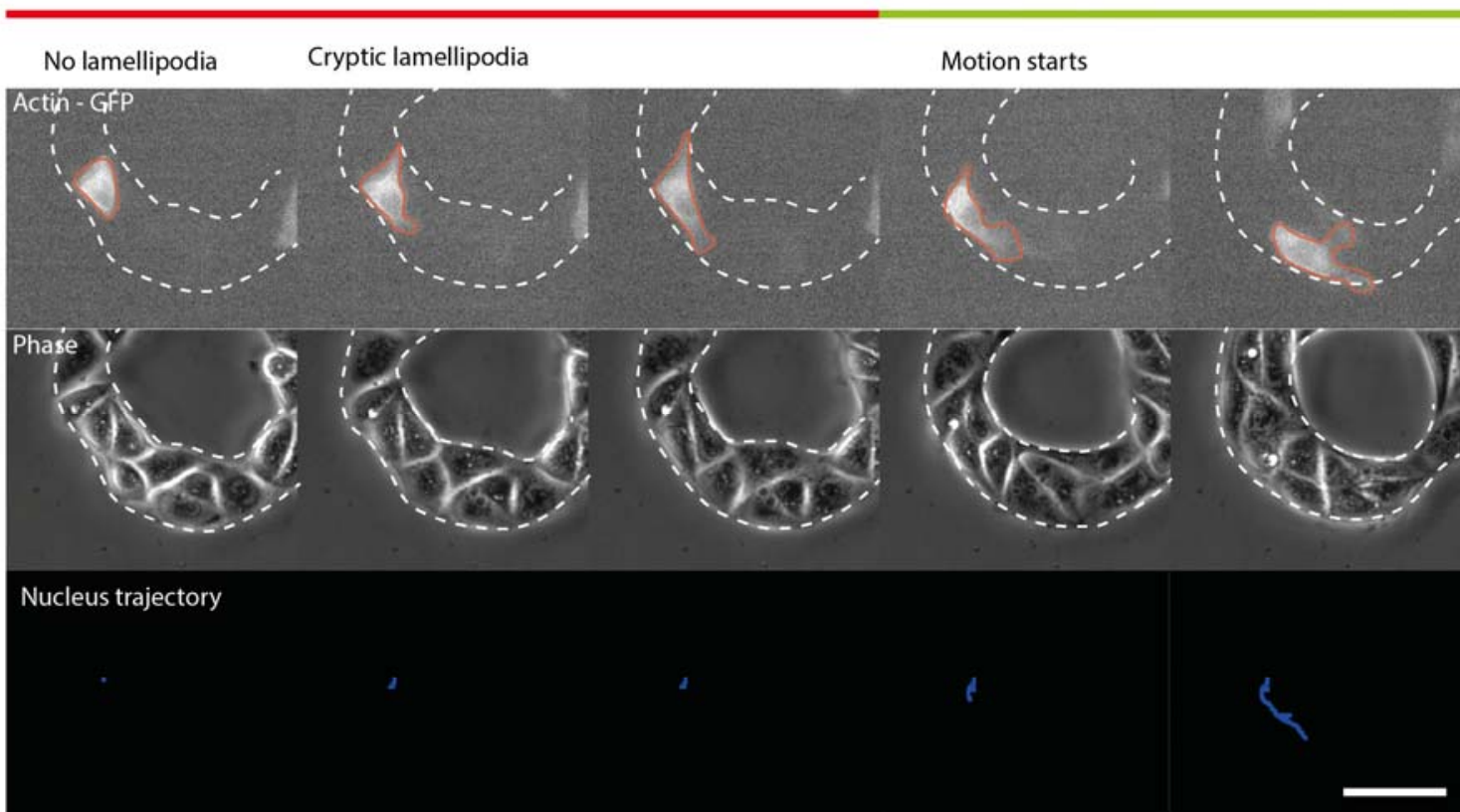

b.
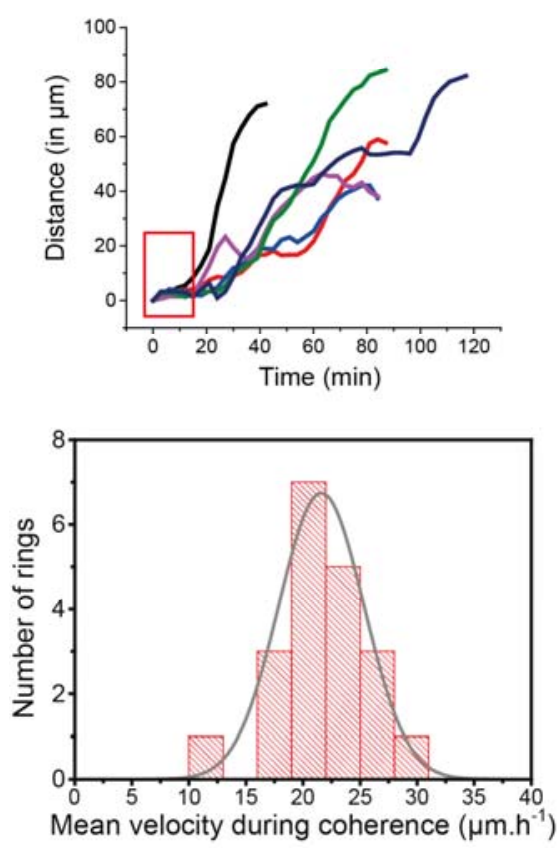

d.

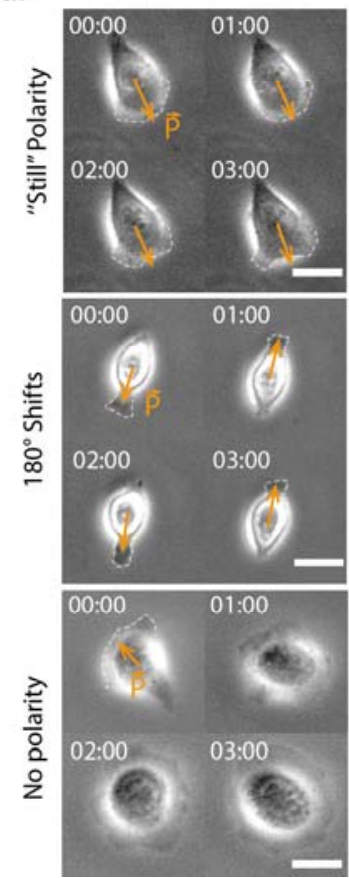

e.

f.
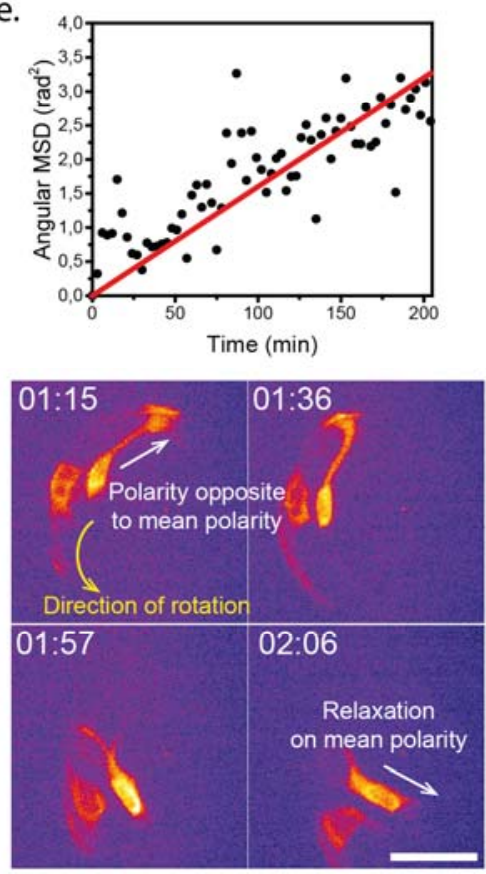

Figure S5. Model characterization. (a) Tracking of cells before lamellipodium growth within the ring. Polarity set by the lamellipodium precedes cell motion as assessed by nuclei displacement shown in (a) and (b). Scale bar $=50 \mu \mathrm{m}$. (c) Distributions of mean ring velocities during coherence. The Gaussian fit sets the values of maximal cell velocities $c$ in the model. (d) Single cell polarity diffusion and 
the associated MSD and diffusion coefficient $D$ shown in (e). Scale bar $=15 \mu \mathrm{m}$. (f) Experimental definition of cell relaxation rate $\mu$. This quantity reflects the velocity at which a given cell polarized in the direction opposite to the mean polarity $\overline{P_{k}}$ relaxes to $\overline{P_{k}}$. Scale bar $=20 \mu \mathrm{m}$.

\begin{tabular}{|c|c|c|c|}
\hline Parameter & Symbol & Value & Units \\
\hline Particle radius & $r$ & 7.5 & $\mu \mathrm{m}$ \\
\hline Velocity & $c$ & 26.92 & $\mu \mathrm{m}^{-1}$ \\
\hline Angular diffusion coefficient & $D$ & 0.96 & $\mathrm{rad}^{2} . \mathrm{h}^{-1}$ \\
\hline Stretching modulus & $k_{s t}$ & $5.10^{3}$ & $\mathrm{pN} . \mu \mathrm{m}^{-1}$ \\
\hline Bending modulus & $k_{b}$ & $10^{3}$ & $\mathrm{pN} . \mu \mathrm{m}$ \\
\hline Relaxation rate on $\overline{P_{k}}$ & $\mu$ & 6 & $\mathrm{rad} . \mathrm{h}^{-1}$ \\
\hline Relaxation rate on $\frac{V_{k}}{\left\|V_{k}\right\|}$ & $v$ & 6 & $\mathrm{rad}^{-1}$ \\
\hline Active force & $f$ & 10 & $\mathrm{nN}$ \\
\hline
\end{tabular}

Table 1. Single cell parameters used in the theoretical model. See also Sec. 1.4 from the Annex. 